\title{
Cell-Scale Modeling to Probe Mechanobiology during Early Cortical Development
}

\author{
Adam Lonnberg ${ }^{1}$, Kara Garcia ${ }^{2}$ \\ ${ }^{1}$ Indiana University School of Medicine, ${ }^{2}$ Indiana University School of Medicine, Department of \\ Radiology and Imaging Sciences
}

Background/Objective: During early cerebral cortex development, neurons form from proliferative glial cells near the ventricular (apical) surface, then migrate along radial glial scaffolds to the cortical surface. In species with wrinkled brains, the presence of basal radial glial cells (bRGCs), radial glial cells which have detached from the ventricular surface, is correlated to the process of gyrification. While mechanical forces are also involved in gyrus creation, the link between the mechanical and biological aspects of this process remains unelucidated. In this study, we hypothesized that radial tension may lead to the production of gyri via the intermediary creation of bRGCs.

Methods: To test this hypothesis, the cell-level modeling software CX3D was used to simulate a system in which radial tension acts on radial glial cells (RGCs), facilitating the semi-stochastic production of bRGCs during the process of neocortex development. The outcome of this model was contrasted with a control case in which bRGCs were not allowed to form, and the two models were compared based upon the presence of neurons on the basal surface.

Results: The production of bRGCs via tension corresponded to a significant increase in the presence of neurons on the pial surface, even if the total number of glial cells-and thus total number of neurons generated-remained constant. Additionally, the likelihood of neurons moving more basally was found to be significantly greater in the presence of bRGCs.

Conclusion and Potential Impact: These results were interpreted to be indications of early gyrus formation. Thus, this study showed that bRGCs-and, ultimately, gyri-may arise from mechanical tension, indicating a possible link between the biological and mechanical explanations of gyrus formation. By providing an alternative lens through which to understand cortical folding, this may have implications for future lines of inquiry, which may expand our understanding of neuro-pathologies associated with misfolding, such as autism and epilepsy. 